\title{
Sammelbericht pro 1889
}

von

J. Gerhardt in Liegnitz.

Die diesjährige Sammelsaison war für die Individuenzahl eine wenig lohnende, für die Artenzahl eine nicht ungünstige, eine Erfahrung, welche vielleicht auch aufserhalb der Provinz stehende Sammler gemacht haben dürften. - Der nachfolgende Bericht umfafst deshalb eine gute Zahl für Niederschlesien wie für Schlesien neuer Arten. Die Namen der letzteren sind gesperrt gedruckt. - L. = Liegnitz. Ortsnamen ohne nähere Angaben beziehen sich auf den Umkreis von Liegnitz. Ueber „Lähn" gilt das im vorjährigen Sammelbericht Gesagte. Einige kritische Staphylinen haben Hrn. Dr. Eppelsheim vorgelegen; ihm dafür hier meinen besonderen Dank.

Dromius nigriventris Th. Unter feuchtem Laube. Berghäuser (Anfang der Vorberge). s. s. (4.)

Hydraena testacea. Bruch b. L. 1 Stück. (Rektor Kolbe.)

Ocalea rivularis. Im Angeschwemmten der Katzbach und des Bobers und unter Laub bei Brechelshof (Jauer), Liegnitz, Lähn. s. s. (7-9.) O. castanea ist $\mathrm{zwar}$ schlesisch, fehlt aber hier gänzlich. Homalota (Atheta) clancula. Unter Laub. Brechelshof. 1 Stück. (Kolbe.) (8.)

H. (Atheta) dilaticornis Kr. Aus Pilzen (Lactarius vellereus, dem grofsen weifsen Reizger mit den weitgestellten Lamellen) gesiebt. Brechelshof (Kolbe 2 q, ich ein $\sigma^{\top}$ ). (10.)

H. (Traumoecia) nigricornis Th. Mit v., aber auch unter Laub. Bis in die Vorberge, doch s. Zuweilen ist das Halssch. der Länge nach sehr breit eingedrückt.

Für Homalota inhabilis (s. D. E. Z. 1888, p. 357) ist daselbst H. (Traumoecia) picipes Th. zu setzen.

H. (Megaloscopa) punctipennis $\mathrm{Kr}$. Unter feuchtem Laube. Berghäuser. $1 \mathrm{Ex}$. (4.)

H. (Anopleta) corvina Th. Mit H. dilatic., nigric., picipennis und vielen gemeinen $\boldsymbol{H}$. in Blätterpilzen, namentl. Anfang Oktbr. hfg. Die Depression auf der Mitte des Halssch. fehlt zuweilen.

H. (Acrotona) parens Rey. 1 Stück aus Pilzen. Vorderhaide.

H. (Acrotona) subsinuata Sahlb. 1 Stück bei L. 
H. (Alaobia) scapularis Sahlb. Von Pflanzen gestrichen. Waltersdorf b. Lähn am Waldessaume.

Oxypoda terrestris $\mathrm{Kr}$. Unter faulendem Laube, im Angeschw. der Katzbach, an unseren Seeen und im Bruch, auch in den Vorbergen. s. s. $(4-7$.) - Dagegen ist O. misella Kr. (vergl. D. E. Z. 1886, p. 220) nicht schlesisch.

Gyrophaena punctulata Muls. (nicht wie bisher puncticollis Th., die nicht schlesisch ist). Fast hfg. in diversen Blätterpilzen, namentlich der Vorberge; oft mit laevipennis und bihamata Th. zusammen.

Quedius nigriceps $\mathrm{Kr}$. In faulenden Pilzen (Lactarius vellereus). Vorderhaide. s. s. (8. 9.) Polkwitz. (Kolbe.)

Qu. obliteratus. Mit v. Vorderhaide, Lähn. (7-9.)

Philonthus addendus Sharp. An Weifsbuchensaft. Vorderhaide, Wasserwald b. Kaltwasser. s. (5.)

Ph. marginatus. Unter Steinen. Hefsberge. (10.)

Sunius immaculatus. Von Pflanzen in d. Vorderhaide. 1 Stück. (6.)

S. pulchellus. Auf den sandigen Pantner Höhen. 2 Stück.

Stenus atratulus. 1 Stück. L. (Kolbe.)

St. cautus. Unt. feucht. Laube. Seeen u. Bahnausstiche. L. s.s. (5.)

St. tempestivus kommt auch mit völlig ausgebildeten Flügeln vor. Ein solches Stück fand Hr. Kolbe im Juli v. J. im Riesengebirge.

Trogophlaeus fuliginosus. Unter feuchtem Laube. Berghäuser. 1 Stück. (4.)

Omalium exiguum. In Pilzen. Vorderhaide. 1 Ex. (9.)

Euplectus sanguineus. Bei Formica rufa. Hummel.

E. signatus. Bei F. nigra. Kaltwasser.

Ptomaphagus longulus. In Pilzen. s. s. Vorderhaide. (9.)

Colon affine. In einem gras- und kräuterreichen, mit einzelnen Laubbäumen durchsetzten Haue bei Vorderhaide. s. (5. 6.)

C. appendiculatum. Ebendort und in den Hefsbergen. s.s. (5.6.)

C. murinum. Im Angeschwemmten der Katzbach. s. s. $(4-6$.

C. denticulatum. Wie C.affine; aufserdem bei Lähn. s.s. (5-7.)

C. bidentatum. Bei Vorderhaide. s. s. (6.)

Liodes (Anisotoma) Triepkei Schmidt. N. s. von Gras in der Nähe von Eichen, gegen Abend, zugleich mit Bradycellus harpalinus Dej., sogar ${ }^{1}$ ) während eines Regens. Vorderbaide. (8. 9.)

L. hybrida Er. Lähn. 1 Stück. (7.)

Agaricophagus cephalotes Schm. Mit v. 1 Stück.

1) Nach Mittheilung des Hrn. Weise begünstigt das Regenwetter das Auftreten der Anisotomen etc. (D. Red.) 
Agathidium polonicum Wank. 1 St. vom Iserkamme. (Kolbe.) (7.)

A. varians Beck. Unter schimmelndem Laube und bei Pilzen. Brechelshof (Kolbe), Vorderhaide.

Trichopteryx cantiana Matth. Mit Tr. Montandoni und sericans. s. Vorderhaide. $(6-9$.

Meligethes subaeneus. Riesengeb. auf Blüthen v. Ranunculus acris.

M. moestus. Hier z. hfg. und nur bis dahin mit M. flavipes vermengt.

Hr. Schilsky vermuthet (D. E. Z. 1889 , p. 340), dafs Cartodere flum Aub. nur in dem Sporenpulver eines Polysaccum lebt; ich habe es im Ustilago verschiedener Pflanzen, namentlich Gramineen meines vor Jahren gesammelten Pilzherbars im Febr. 1883 in ungezählten Mengen beobachtet. Das Thier scheint überhaupt Staubund Brandpilze zu lieben.

Corticaria distinguenda. Auf niederen Pflanzen, bisweilen mit C. transversalis Gyll. Kaltwasser, Vorderhaide, Hefsberge, Lähn. z. s. $(4-6$.)

Attagenus piceus ist etwas seltener als A. marginicollis Küst.

Curimus murinus wird zwar von Letzner als Bürger der Hefsberge aufgeführt, wahrscheinlich aber liegt eine Verwechselung mit dem daselbst von mir beobachteten C. Erichsoni zu Grunde. C. murinus wurde erst in diesem Jahre von Hrn. Kolbe am ausfliefsenden Safte einer Weifsbuche bei Vorderhaide aufgefunden.

Cyphon palustris. An Sumpfstellen des Vorgebirges z.s. Lähn, Hefsb. (6-8.)

Cantharis frbulata. Mitten im Walde nördlich Vorderhaide von Pteris aquilinum. 1 Stück. (6.)

Malthodes mysticus Ksw. Lähn. s. (7.)

M. trifurcatus Ksw. 1 Stück wahrscheinl. aus dem Riesengeb.

Drilus concolor. Lähn. 1 Stück, mit Rhagonycha atra gestrichen.

Julistus memnonius. Auf blühenden Kiefern. Panthener Höhen. s. s. Dagegen fehlt hier J.floralis, den Hr. Kolbe bei Maltsch a. O. fand. Opilo pallidus. Von einer alten Eiche geklopft. Kaltwasser. 1 Ex. (6.)

Ernobius abieticola. Riesengebirge. 1 Stück. (7.) (Kolbe.)

E. abietis F. Vorderbaide. 1 Stück. 8.

E. anabaptista Gozis. Ich besitze von dieser Art zwei in Niederschlesien von mir gesammelte Stücke.

Xyletinus laticollis. In Hauen. Vorderhaide, Lähn. Häufiger als ater. (6.) 
Xylophilus nigrinus. Hfg. Ende Mai gegen Abend (bald nach Sonnenuntergang am zahlreichsten) auf etwa zweijährigen Kieferpflanzen bei Vorderhaide.

Anaspis ruficollis F. sitzt gern unter den schattenspendenden Wedeln von Pteris aquilinum mitten im Walde, weshalb man ihn in der Sonne, wenigstens bei L., selten streicht.

Liosoma ovatulum var. Discontignyi Bris. Unter Laub. 1 Stück. Berghäuser. (4.)

Hypera tesselata Hbst. Zur Blüthezeit des Orobus niger von Kräutern gestr. Hefsberge, Lähn. z. s. $(5-6$.)

Anthonomus varians var. perforator Hbst. Von dieser für Schlesien seltenen Var. fand sich 1 Stück aus Niederschlesien in meiner Sammlung bei Anthonomus rubi.

A.? spec. Lähn, v. Prunus padus. Die Bestimmung dieser für Deutschland vielleicht neuen Art dürfte durch Desbroches erfolgen.

Rhyncolus nitidipennis Thoms. In einem Hau bei Vorderhaide. 1 Stück. (5.)

Grammoptera analis. Vorderhaide. (Kolbe.) 1 Stück.

Lina 20 -punctata. Fasanenbusch bei Brechelshof unter Laub. (8.) (Kolbe.)

Scymnus impexus Muls. Auf der Wintereiche (Quercus sessiliflora). Vorderhaide, Hefsberge. z. s.

\section{Ueber Homalota truncata Epp.}

In der Stett. ent. Zeitung 1875, p. 362 habe ich als neue Art nach einem einzigen, noch dazu schadhaften und abgeriebenen böhmischen Stücke eine Homalota truncata aufgestellt, deren Beschreibung, nachdem mir in letzter Zeit aus anderer Gegend reicheres Material zugegangen ist, in einigen Stücken corrigirt und ergänzt werden mufs. Es steht diese Art sowohl bezüglich ihrer allgemeinen Körperform, wie hinsichtlich der Ausbildung der männlichen Geschlechtsmerkmale in der allerinnigsten Beziehung zu Taxicera sericophila und deplanata, sie hat, wie diese, gerandete Schläfen, und mufs denselben systematisch angereiht werden, wiewohl sie nicht eigentlich zur Untergattung Taxicera gehört, da ihr die perfoliirten Fühler fehlen, während andererseits ihre Fühlerbildung, wenigstens im weiblichen Geschlechte, mit derjenigen der beiden Taxicera-Arten das Gemeinschaftliche hat, dafs das 5te Glied doppelt 


\section{$2 \mathrm{BHL}$ Biodiversity Heritage Library}

Gerhardt, Julius. 1890. "Sammelbericht pro 1889." Deutsche entomologische Zeitschrift 1890(3), 200-203. https://doi.org/10.1002/mmnd.48018900326.

View This Item Online: https://www.biodiversitylibrary.org/item/103589

DOI: https://doi.org/10.1002/mmnd.48018900326

Permalink: https://www.biodiversitylibrary.org/partpdf/235480

\section{Holding Institution}

Harvard University, Museum of Comparative Zoology, Ernst Mayr Library

\section{Sponsored by}

Harvard University, Museum of Comparative Zoology, Ernst Mayr Library

\section{Copyright \& Reuse}

Copyright Status: Public domain. The BHL considers that this work is no longer under copyright protection.

This document was created from content at the Biodiversity Heritage Library, the world's largest open access digital library for biodiversity literature and archives. Visit BHL at https://www.biodiversitylibrary.org. 